Aim of the study: To assess the impact of the COVID-19 pandemic on the diagnosis and treatment of patients at a tertiary hospital in Poland.

Material and methods: This was a retrospective review of head and neck cancer patients who presented to the multidisciplinary tumour board (MTB) during the 12-month period from March 2020 to February 2021 and compared to patients who presented to the MTB during the prior, pre-pandemic 12 -month period from February 2019 to March 2020: Patient demographic and clinical variables were compared: sex, age at diagnosis, distance from hospital, date of first visit, radiological diagnosis, pathology specimen, MTB meeting, and initiation of primary and adjuvant treatment Results: The number of patients who presented to the MTB increased by $22 \%$ (278 to 340 ) from the pre-pandemic to the pandemic period. The mean time from MTB presentation to treatment initiation increased sig nificantly from 17.1 to 21.7 days. The mean time from first visit to treatment start increased from 44.7 to 54.4 days. The proportion of patients with early-stage oropharyngeal cancer who underwent primary surgery rose from $47.3 \%$ to $86.6 \%$. The percentage of patients who received palliative radiotherapy increased from $20.5 \%$ to $32.9 \%$. The proportion of patients who received best supportive care rose from $1.8 \%$ to $6.2 \%$.

Conclusions: One of the most notable findings of this study was the increased time from first visit to treatment initiation, which could negatively impact patient outcomes. The differences in the treatment received in these two periods should be further evaluated to determine their influence on survival.

Key words: COVID-19, head neck, pandemic, oncology, impact.

Contemp Oncol (Pozn) 2021; 25: 264-269 DOI: https://doi.org/10.5114/wo.2021.111310

\section{The impact of the COVID-19 pandemic on the management of head and neck cancer patients at a tertiary care institution in Poland}

\author{
Mateusz Szewczyk ${ }^{1}$, Jakub Pazdrowski ${ }^{1}$, Paweł Golusiński², \\ Paweł Pazdrowski ${ }^{3}$, Barbara Więckowska ${ }^{4}$, Wojciech Golusiński ${ }^{1}$
}

${ }^{1}$ Department of Head and Neck Surgery, Poznan University of Medical Sciences, Poznan, Poland

2Department of Otolaryngology and Maxillofacial Surgery, University of Zielona Gora, Poland

${ }^{3} \mathrm{Head}$ and Neck Surgery Student Group, Department of Head and Neck Surgery, Poznan University of Medical Sciences, Poznan, Poland

${ }^{4}$ Department of Computer Science and Statistics, Poznan University of Medical Sciences, Poznan, Poland

\section{Introduction}

In December 2019, the first patient was diagnosed with severe acute respiratory syndrome coronavirus disease (SARS-Cov-2), eventually leading to a global pandemic. The clinical manifestations of coronavirus disease 2019 (COVID-19) can vary widely, ranging from mild respiratory symptoms to severe viral pneumonia, which may be followed by respiratory failure and death [1]. Shortly after the emergence of the virus, treating patients with COVID-19 became a key priority and severe restrictions were put in place, thus limiting health care resources, even in hospitals that do not treat these patients. Unsurprisingly, these restrictions also affected the care of cancer patients in terms of access to diagnosis and treatment [2].

In this context, the aim of the study was to assess the impact of COVID-19 on the diagnosis and treatment of patients at the Greater Poland Cancer Center (GPCC), a comprehensive cancer care centre. We compared the characteristics of patients diagnosed with head and neck cancer during the 12-month, pre-pandemic period prior to implementation of pandemic-related restrictions in Poland in March 2020 to patients diagnosed and treated during the pandemic period (March 2020 to February 2021). More specifically, we sought to determine whether there were any differences between the two periods in terms of demographic and clinical variables, and the impact of the pandemic on the diagnosis and treatment of these patients.

\section{Material and methods}

This was a retrospective review of all head and neck cancer patients who presented to the multidisciplinary tumour board (MTB) during the 12-month period from March 1, 2020 to February 28, 2021. This patient group was compared to patients who presented to the MTB during the prior, pre-pandemic 12-month period (February 1, 2019 to February 28, 2020).

Patients who had received any prior head and neck treatment at the GPCC were excluded from the analysis due to the risk of bias as these patients would have had greater access than newly diagnosed patients to the outpatient department. Patients with thyroid cancer were also excluded from the study to avoid influencing the study results given that the preoperative assessment and post-operative treatment of these patients are performed at other treatment centres (not at the GPCC). 
Multidisciplinary tumour board meetings were held weekly during the pandemic via teleconference, but otherwise the decision-making process remained the same as during the pre-pandemic period. Patient demographic and clinical variables evaluated included sex, age at diagnosis, distance from the GPCC, and dates of the following: first visit, radiological diagnosis, pathology specimen, MTB meeting, and initiation of primary and adjuvant treatment, and centre where radiological and histopathologic diagnostics were performed (GPCC or other). Tumour characteristics, including primary site, TNM status ( $T$ - size of the tumour and any spread of cancer into nearby tissue, $\mathrm{N}$ - spread of cancer to nearby lymph nodes, $\mathrm{M}$ - metastasis) $-8^{\text {th }}$ edition, and nodal status were recorded. Primary treatment was categorised as radical-intent (surgery, radiotherapy, and/or induction chemotherapy) or palliative treatment (radiotherapy and/or best supportive care).

Due to the retrospective nature of the study, the approval of the Research Ethics Board at the local University of Medical Sciences was not considered necessary.

\section{Statistical analysis}

The comparative analysis of the quantitative data was performed with Student's t-test for independent groups (unpaired t-test) or the U Mann-Whitney test if the normality assumption was not met. The Kolmogorov-Smirnov test was used to evaluate the normality of the distribution. Dichotomous variables were compared with the $\chi^{2}$ test when Cochran's condition was met or Fisher's exact test when it was not met. To compare multiple proportions within one hypothesis, the test for one proportion was used.

\section{Results}

A total of 278 patients were discussed at MTB meetings in the pre-pandemic period versus 340 patients during the pandemic, an increase of $22 \%$. No difference was found between the percentage of male patients, mean patient age, or place of residence (Wielkopolska province or elsewhere) (Table 1).

Similarly, no differences were observed in the percentage of patients receiving radiological diagnosis at the GPCC (49\% vs. 50\%). However, the proportion of patients whose histopathologic evaluation of the biopsied tumour was performed at the centre increased from $48.6 \%$ in the pre-COVID period to $57.4 \%(p=0.029)$ (Table 1$)$.

The mean time from first visit to the MTB meeting differed slightly (but not significantly) from the pre-pandemic to the pandemic period (27.9 vs. 33.5 days, respectively) The mean time from presentation at the MTB to treatment initiation increased significantly from 17.1 to 21.7 days $(p=0.006)$. Similarly, the mean time from first visit to treatment start also increased significantly (44.7 vs. 54.4 days, $p=0.004)$ (Table 1).

The percentage of oral cancer patients decreased significantly from $45.3 \%$ to $32.1 \%$ ( $p=0.01)$ while the proportion of nasopharyngeal cancer patients rose from $0.4 \%$ to $3.2 \%(p=0.01)$. There were no significant differences between the two time periods in the overall percentage of locally advanced (stage T3-T4) cases (54\% vs. 53\%), nor by tumour location (i.e., oral cancer, nasopharyngeal cancer).

No differences were observed in the percentage of patients with nodal involvement ( $\mathrm{N}+$ disease), which increased slightly (not significantly) from 51.5\% to 55.6\%. However, the proportion of patients with nasopharyngeal cancer and $\mathrm{N}+$ disease was significantly higher in the COVID-19 period (0.7\% vs. $5.3 \%, p=0.038)$. The percentage of patients with advanced disease (stage T3-T4) at diagnosis was comparable $(67.5 \%$ vs. $72.6 \%$, respectively) (Table 2 ).

No significant differences were detected in the percentage of patients undergoing surgery (71\% vs. $75.4 \%)$, nor were any differences observed for this same variable by cancer site: in patients with early-stage laryngeal cancer, $80.5 \%$ underwent surgery in the pre-pandemic period vs. $74 \%$ during the pandemic. By contrast, for patients diagnosed with early-stage oropharyngeal cancer, the percentage of patients who underwent primary surgery increased significantly from $47.3 \%$ to $86.6 \%(p=0.001)$ (Table 3$)$.

The percentage of patients who received palliative radiotherapy increased significantly, from $20.5 \%$ to $32.9 \%$ ( $p=0.001$ ). In addition, the proportion of patients who received best supportive care also increased significantly, from $1.8 \%$ to $6.2 \%$ ( $p=0.007$ ).

Themeantime from surgery to the start of adjuvantradiotherapy remained essentially unchanged ( 57.5 vs. 56.3 days) (Table 3). Regarding primary and adjuvant radiotherapy, prolonged overall treatment time (OTT) was observed, due

Table 1. Patient demographic characteristics and time frames for disease diagnosis and treatment

\begin{tabular}{|c|c|c|c|}
\hline & $\begin{array}{c}\text { Pre-pandemic, } \\
n(\%) \\
N=278\end{array}$ & $\begin{array}{c}\text { COVID-19 period, } \\
n(\%) \\
N=340\end{array}$ & $p$-value \\
\hline Sex, males & $206(74.1)$ & $263(77.4)$ & 0.347 \\
\hline Mean age at diagnosis (years) & 63.6 & 64.1 & 0.460 \\
\hline Place of residence, Wielkopolska province & $226(81.3)$ & $287(84.4)$ & 0.305 \\
\hline Radiological diagnosis at the GPCC & $136(48.9)$ & $170(50)$ & 0.790 \\
\hline Tumor biopsy at the GPCC & $135(48.6)$ & $195(57.4)$ & 0.029 \\
\hline Mean time from first visit to MTB (days) & 27.9 & 33.5 & 0.105 \\
\hline Mean time from MTB to treatment start (days) & 17.1 & 21.7 & 0.006 \\
\hline Mean time from first visit to treatment initiation (days) & 44.7 & 54.4 & 0.004 \\
\hline Mean waiting time from surgery to adjuvant radiotherapy (days) & 57.5 & 56.3 & 0.693 \\
\hline
\end{tabular}


Table 2. Tumour location and disease stage

\begin{tabular}{|c|c|c|c|}
\hline & $\begin{array}{c}\text { Pre-pandemic period, } \\
n(\%) \\
N=278\end{array}$ & $\begin{array}{c}\text { COVID-19 period, } \\
n(\%) \\
N=340\end{array}$ & $p$-value \\
\hline Larynx/hypopharynx & $89(32)$ & $113(33.2)$ & 0.845 \\
\hline Oral cavity & $126(45.3)$ & $109(32.1)$ & 0.010 \\
\hline Oropharynx & $39(14)$ & $64(18.8)$ & 0.174 \\
\hline Salivary glands & $8(2.9)$ & $12(3.5)$ & 0.829 \\
\hline CUP & $7(2.5)$ & $18(5.3)$ & 0.128 \\
\hline Nasal cavity/sinuses & $8(2.9)$ & $13(3.8)$ & 0.683 \\
\hline Nasopharynx & $1(0.4)$ & $11(3.2)$ & 0.017 \\
\hline Locally advanced disease (T1-T2) & $150(54)$ & $181(53.2)$ & 0.858 \\
\hline Regional disease $(\mathrm{N}+)$ & $143(51.4)$ & $189(55.6)$ & 0.303 \\
\hline Advanced stage (III/IV) & $187(67.5)$ & $247(72.6)$ & 0.165 \\
\hline
\end{tabular}

CUP - carcinoma of unknown primary

Table 3. Treatment type characteristics

\begin{tabular}{|c|c|c|c|}
\hline & $\begin{array}{c}\text { Pre-pandemic period, } \\
\qquad \begin{array}{c}n(\%) \\
N=278\end{array}\end{array}$ & $\begin{array}{c}\text { COVID-19 period, } \\
n(\%) \\
N=340\end{array}$ & $p$-value \\
\hline Primary surgical treatment & $157(71)$ & $172(75.4)$ & 0.337 \\
\hline Primary surgical treatment in early stage (T1-T2) laryngeal cancer & $29(80.5)$ & $40(74)$ & 0.613 \\
\hline Primary surgical treatment in early stage (T1-T2) oropharyngeal cancer & $9(47.3)$ & $26(86.6)$ & 0.001 \\
\hline Palliative radiotherapy & $57(20.5)$ & $112(32.9)$ & 0.001 \\
\hline Ineligible for treatment (best supportive care only) & $5(1.8)$ & $21(6.2)$ & 0.007 \\
\hline
\end{tabular}

to COVID-related gaps in radiation therapy. Mean interruption time was 20 days. In all cases radiotherapy and chemotherapy were adapted after the gap, including additional fractions of radiotherapy. To shorten the OTT in order to decrease the probability of treatment interruption, hypofractionated schedules of irradiation were used, where clinically justified. On the other hand, the COVID pandemic has not affected the decision-making process in induction chemotherapy application (5 patients in pre-COVID and 7 patients in COVID period).

\section{Discussion}

Our study is the first to show the impact of the COVID-19 pandemic on head and neck patients over the course of a full 12-month time period. The mean time from MTB presentation to treatment initiation increased from 17.1 to 21.7 days ( $p=0.006$ ), as did the mean time from first visit to treatment start (from 44.7 to 54.4 days, $p=0.004)$. This significant increase in the time from diagnosis to treatment could have negative implications for survival outcomes, although we did not find any significant differences between the time periods in terms of disease staging.

\section{Impact of general restrictions on medical care}

The COVID-19 pandemic has influenced every area of medicine. One of the major impacts has been restricted access to medical care [3]. In Poland, similar to other countries, patients' access to general practitioners during the pandemic was limited and most consultations were conducted through telemedicine, which has a higher risk of an incorrect diagnosis [4]. Many hospitals around the world have been transformed into COVID-19 treating institutions, which means that patients are less likely to be accurately diagnosed and treated in a timely manner when the disease is still in early stages [5]. Nevertheless, cancer centres in many countries have remained open, with adequate local restrictions, while other hospitals were responsible for treating only patients with COVID-19 [6]. In fact, this approach allowed cancer centres in those countries to treat more patients during the pandemic than in the prior year $[5,7]$. We observed similar results, as evidenced by the $22 \%$ increase in the number of patients who presented to the MTB. In Poland, as in other countries, many hospitals were transformed into COVID-treating institutions, but this did not affect many cancer centres, including ours. Outpatient visits decreased by $50 \%$ from March to June 2020 and by $25 \%$ thereafter. Even so, most patients were able to schedule a consultation and receive a treatment proposal. By contrast, some high volume cancer centres experienced a substantial reduction in patients in the most acute phase of the pandemic, with one centre (MD Anderson) in the United States of America reporting a $25 \%$ decrease over a 6 -week period [8].

In the context of limited access to medical care due to pandemic-related restrictions, the distance from the patient's home to the hospital could be an important factor. To evaluate the impact of distance, we divided our patients into two groups: those living in the same province (Wielkopolska) as the hospital and those residing outside 
of this province. That analysis showed that the percentage of patients from the relatively large Wielkopolska province (30 $000 \mathrm{~km}^{2}$ ) remained essentially unchanged from the pre-pandemic period ( $81 \%$ vs. $84 \%$, respectively). To our knowledge, the only other study to examine this issue was performed by Kiong et al. at the MD Anderson Cancer Center, who did not find any significant differences either [8].

In terms of access to cancer diagnosis and treatment, we found that radiologic tests performed at our centre were similar in the two groups (49\% vs. 50\%), although a significantly higher proportion of patients (57\% vs. 48.6\%) required a biopsy during the COVID-19 period. This finding may be related to the need for qualified staff and appropriate equipment to perform laryngeal and hypopharyngeal tumour biopsies. These procedures are usually done under general anaesthesia, thus limiting the role of outside centres. We were unable to identify any other studies that have evaluated this variable to compare results.

\section{Demographic data and tumour location}

Most of the studies that have compared patients treated in the pre-pandemic and pandemic periods have not found any significant demographic differences in sex or age, similar to our results $[7,9,10]$. However, we did find a significantly higher proportion of patients diagnosed with oral cancer in the pre-COVID period ( $45.3 \%$ vs. $32.1 \%$, Table 2 ), but the reason for this difference is difficult to determine. Kiong et al. found no significant differences in the main head and neck cancer locations at their centre between the two periods; nor did they find any other studies reporting such differences [8]. In contrast, Thomson and colleagues found a substantial increase in the percentage of patients diagnosed with oral cancer (from 23\% to 44.4\%), but they did not explain the potential reasons for this difference [7]. Interestingly, we observed a significant increase in the number of nasopharyngeal cancer patients (from 1 to 11), perhaps because most of the symptoms and side effects of COVID-19 affect the nasal cavity and paranasal sinuses, which could have led to early detection of nasopharyngeal cancer during the diagnostic process for COVID-19. Nonetheless, these results need further research.

\section{Diagnostic and treatment time frames}

Early diagnosis is a crucial variable in the odds of treatment success in cancer patients, with numerous studies showing that diagnostic delays negatively impact survival outcomes. Rygalski et al. retrospectively evaluated 37730 patients with head and neck cancer included in the National Cancer Database (NCD), finding that the cut-off point for time to surgery that had the greatest impact on survival was 67 days [11]. In another study, Murphy et al. evaluated data from 51655 patients included in the NCD who received curative-intent treatment for oral, oropharyngeal, or hypopharyngeal cancer, finding that time from diagnosis to treatment $>60$ days was consistently associated with a higher risk of death [12]. In our study, we found a small but non-significant increase in time from first visit to the MTB meeting (27.9 vs. 33.5 days), in line with the data reported by Kiong et al. [8]. We also observed a significant increase in time (from 17.1 to 21.7 days) from presentation to the MTB to treatment initiation, a finding that is consistent with the data described by Tevetoğlu et al. [10]. Similarly, the time from the first visit to treatment initiation also increased significantly in our study (44.7 vs. 54.4 days), a finding that contrasts with Tevetoğlu et al., who found no significant changes (Kiong et al. did not evaluate this variable).

Our data show that the percentage of radiological diagnoses performed at the GPCC was similar in both periods. By contrast, a significantly greater percentage of patients underwent biopsy in the COVID period ( $57.4 \%$ vs. $48.6 \%$ in the pre-pandemic period). These data show that although restrictions in non-cancer centres at least partially affected outpatient diagnostic tests, they had no significant impact on the time interval to the MTB meeting. However, waiting times for inpatient treatment differed between the periods, and the restrictions in place at our centre and other centres could have affected the time from MTB to treatment $[13,14]$. Another factor that may have influenced the time to treatment initiation is patient-related concerns about entering the hospital during the pandemic [15].

\section{Type of treatment}

Several groups developed recommendations on the management of patients with head and neck cancer during the pandemic, including general recommendations as well as specific surgical and non-surgical recommendations [16-20]. Treatment of head and neck cancer has been restricted around the world due to capacity limitations and to the increased risk of infection for both staff and patients. In our centre, the intensive care unit (ICU) had only limited restrictions; therefore the decision-making process for surgical treatment was not influenced by any ICU-related restrictions. With regard to non-surgical treatment, we observed some delays during the 12-month COVID-19 study period related to inpatient coronavirus infections during the course of radiotherapy, which perhaps explains why there was no significant difference between the two periods in the proportion of patients eligible for radical surgery (71\% vs. $75.4 \%$ ). Kiong and colleagues did not observe any difference in surgical vs. non-surgical treatment, although fewer patients were considered eligible for primary surgery than in our study (47.3\% vs. $73.2 \%$ ).

We also assessed the type of treatment in two specific anatomic locations (larynx and oropharynx), which were selected because oncological outcomes for these two sites are similar regardless of the treatment type in patients with early-stage disease [21-25]. We found no significant difference between the pre-COVID and COVID periods in terms of the proportion of laryngeal cancer patients treated surgically ( $80 \%$ vs. $74 \%$, respectively), but we did find a significant difference in early-stage oropharyngeal cancer ( $47 \%$ vs. $86 \%$ ), perhaps due to the use of minimally invasive surgery (mainly robotic surgery) in these patients, where the risk of tracheostomy is low and the hospital stay is much shorter than in radical radiotherapy.

Our data show that a significantly higher proportion of patients received palliative radiotherapy during the pandemic period (20.5\% vs. $32.9 \%$ ) and palliative care alone was indicated in a higher percentage of patients (1.8\% vs. $6.2 \%)$. Both 
findings are likely directly related to the pandemic. Although disease severity (TNM staging) did not differ between the two periods, the limited access to basic medical care (with the consequent delays in diagnosis and treatment) resulted in an increase in the number of patients ineligible for radical treatment due to comorbidities and cancer-related malnutrition. Of the 21 patients in the COVID-19 period referred to best supportive care, six were offered palliative radiotherapy but declined due to pandemic-related fear. Given the importance of palliative care to ensure adequate pain management and nutritional and respiratory support, we believe that a symptom-based approach to these patients should be taken during the pandemic. In this regard, Singh and colleagues published recommendations on the management of palliative patients during the pandemic, emphasizing the need for better access to drugs, greater use of teleconsultation, and wider community support [26].

\section{Strengths and limitations of the study}

The main study limitation is the retrospective design. Another limitation is that we did not examine symptom duration prior to the first visit, mainly due to missing data. Although there were some restrictions in outpatient visits and delays in the start of radiotherapy, none of the units had to be closed for more than 2-3 days and none of the doctors had to be transferred to other hospitals to treat COVID-19 patients. A final limitation is that we did not assess or compare patient outcomes. By contrast, an important strength of this study is that it is, to our knowledge, the first to examine the effect of the pandemic on patients with head and neck cancer during an extended time period (12 months) including several waves of the pandemic.

\section{Conclusions}

The COVID-19 pandemic has had a major impact on the medical care of patients with head and neck cancer in terms of outpatient diagnosis and inpatient treatment. The results of this study suggest that, in the context of pandemic-related restrictions imposed in non-oncological hospitals, specialized cancer centres must be prepared to consult, diagnose, and treat greater numbers of patients. The increased time from first visit to treatment initiation observed in this study (and others) suggests that greater efforts need to be made to avoid diagnostic delays. Differences in the treatment recommendations between the two time periods should be evaluated in future studies to determine how this affects survival curves. Finally, it would be valuable to determine whether the significant increase in the number of patients with nasopharyngeal cancer is virus-related.

The authors declare no conflict of interest.

\section{References}

1. Xu H, Zhong L, Deng J, Peng J, et al. High expression of ACE2 receptor of 2019-nCoV on the epithelial cells of oral mucosa. Int J Oral Sci 2020; 12: 8.
2. Shaw R, COVIDSurg Collaborative. UK Head and neck cancer surgical capacity during the second wave of the COVID-19 pandemic: have we learned the lessons? COVIDSurg collaborative. Clin Otolaryngol 2021; 46: 729-735.

3. De Felice F, Polimeni A, Tombolini V. The impact of Coronavirus (COVID-19) on head and neck cancer patients' care. Radiother Oncol 2020; 147: 84-85.

4. Omboni S. Telemedicine during the COVID-19 in Italy: a missed opportunity? Telemed J E Health 2020; 26: 973-975.

5. Salzano G, Maglitto F, Guida A, et al. Surgical oncology of the head and neck district during COVID-19 pandemic. Eur Arch Otorhinolaryngol 2021; 278: 3107-3111.

6. Nowara E, Działach E, Grajek M, et al. Outcome of COVID-19 infection in cancer patients during active systemic anticancer treatment. Single-institution experience. A retrospective analysis. Contemp Onkol 2021; 25: 147-152.

7. Thompson JA, Lubek JE, Amin N, et al. Impact of the novel coronavirus 2019 (COVID-19) pandemic on head and neck cancer care. Otolaryngol Neck Surg 2021; 019459982110045.

8. Kiong KL, Diaz EM, Gross ND, et al. The impact of COVID-19 on head and neck cancer diagnosis and disease extent. Head Neck 2021; 43: 1890-1897.

9. Laccourreye O, Mirghani H, Evrard D, et al. Impact of the first month of COVID-19 lockdown on oncologic surgical activity in the Île-de-France region university hospital otorhinolaryngology departments. Eur Ann Otorhinolaryngol Head Neck Dis 2020; 137: 273-276.

10. Tevetoğlu F, Kara S, Aliyeva C, et al. Delayed presentation of head and neck cancer patients during COVID-19 pandemic. Eur Arch Otorhinolaryngol 2021; 270: 5081-5085.

11. Rygalski CJ, Zhao S, Eskander A, et al. Time to surgery and survival in head and neck cancer. Ann Surg Oncol 2021; 28: 877-885.

12. Murphy CT, Galloway TJ, Handorf EA, et al. Survival impact of increasing time to treatment initiation for patients with head and neck cancer in the United States. J Clin Oncol 2016; 34: 169-178.

13. Malicki J, Martenka P, Dyzmann-Sroka A, et al. Impact of COVID-19 on the performance of a radiation oncology department at a major comprehensive cancer centre in Poland during the first ten weeks of the epidemic. Rep Pract Oncol Radiother 2020; 25: 820-827.

14. Gupta A, Arora V, Nair D, et al. Status and strategies for the management of head and neck cancer during COVID-19 pandemic: Indian scenario. Head Neck 2020; 42: 1460-1465.

15. Sutcuoglu O, Yazici O, Ozet A, Ozdemir N. Harmful consequences of COVID-19 fear in patients with cancer. BMJ 2020: bmjspcare-2020-002628.

16. Baird BJ, Sung CK. Coronavirus disease-19. Otolaryngol Clin North Am 2020; 53: 1159-1170.

17. Mehanna H, Hardman JC, Shenson JA, et al. Recommendations for head and neck surgical oncology practice in a setting of acute severe resource constraint during the COVID-19 pandemic: an international consensus. Lancet Oncol 2020; 21: e350-e359.

18. Salari A, Jalaeefar A, Shirkhoda M. What is the best treatment option for head and neck cancers in COVID-19 pandemic? A rapid review. Am J Otolaryngol 2020; 41: 102738.

19. Mireștean CC, Crișan A, Mitrea A, et al. New challenges of treatment for locally advanced head and neck cancers in the COVID-19 pandemic era. J Clin Med 2021; 10: 587.

20. Jeannon J-P, Simo R, Oakley R, et al. Head and neck cancer surgery during the coronavirus pandemic: a single-institution experience. J Laryngol Otol 2021; 135: 168-172.

21. Baird BJ, Sung CK, Beadle BM, Divi V. Treatment of early-stage laryngeal cancer: a comparison of treatment options. Oral Oncol 2018; 87: 8-16.

22. Howard J, Masterson L, Dwivedi RC, et al. Minimally invasive surgery versus radiotherapy/chemoradiotherapy for small-volume primary oropharyngeal carcinoma. Cochrane Database Syst Rev 2016; 12: CD010963.

23. Mourad M, Dezube A, Moshier E, Shin E. Geographic trends in management of early-stage laryngeal cancer: trends in laryngeal cancer management. Laryngoscope 2016; 126: 880-884. 
24. Pedro C, Mira B, Silva P, et al. Surgery vs. primary radiotherapy in early-stage oropharyngeal cancer. Clin Transl Radiat Oncol 2018; 9: 18-22.

25. Stelmes J-J, Gregoire V, Poorten VV, et al. Organ preservation and late functional outcome in oropharyngeal carcinoma: rationale of EORTC 1420, the "best of" trial. Front Oncol 2019; 9: 999.

26. Singh AG, Deodhar J, Chaturvedi P. Navigating the impact of COVID-19 on palliative care for head and neck cancer. Head Neck 2020; 42: 1144-1146.

\section{Address for correspondence}

Mateusz Piotr Szewczyk, PhD

Department of Head and Neck Surgery

Poznan University of Medical Sciences

Poznań, Poland

e-mail:mateuszszewczyk@yahoo.pl

Submitted: 29.10 .2021

Accepted: 09.11.2021 